\title{
Control and Characterization of Intramolecular Dynamics with Chirped Femtosecond Three-Pulse Four-Wave Mixing
}

\author{
Igor Pastirk, ${ }^{\dagger}$ Vadim V. Lozovoy, ${ }^{\ddagger}$ Bruna I. Grimberg, Emily J. Brown, and Marcos Dantus* \\ Department of Chemistry and Center for Fundamental Materials Research, Michigan State University, \\ East Lansing, Michigan 48824
}

Received: June 15, 1999; In Final Form: August 23, 1999

\begin{abstract}
Experimental control and characterization of intramolecular dynamics are demonstrated with chirped femtosecond three-pulse four-wave mixing (FWM). The two-dimensional (spectrally dispersed and timeresolved) three-pulse FWM signal is shown to contain important information about the population and coherence of the electronic and vibrational states of the system. The experiments are carried out on gas-phase $\mathrm{I}_{2}$ and the degenerate laser pulses are resonant with the $\mathrm{X}$ (ground) to B (excited) electronic transition. In the absence of laser chirp, control over population and coherence transfer is demonstrated by selecting specific pulse sequences. When chirped lasers are used to manipulate the optical phases of the pulses, the two-dimensional data demonstrate the transfer of coherence between the ground and excited states. Positive chirps are also shown to enhance the signal intensity, particularly for bluer wavelengths. A theoretical model based on the multilevel density matrix formalism in the perturbation limit is developed to simulate the data. The model takes into account two vibrational levels in the ground and the excited states, as well as different pulse sequences and laser chirp values. The analytical solution allows us to predict particular pulse sequences that control the final electronic state of the population. In a similar manner, the theory allows us to find critical chirp values that control the transfer of vibrational coherence between the two electronic states. Wave packet calculations are used to illustrate the process that leads to the observation of ground-state dynamics. All the calculations are found to be in excellent agreement with the experimental data. The ability to control population and coherence transfer in molecular systems is of great importance in the quest for controlling the outcome of laser-initiated chemical reactions.
\end{abstract}

\section{Introduction}

Experimental demonstrations of laser control of chemical reactions in the past decade ${ }^{1-3}$ have solidified this field of research and garnered great excitement. While the field is quickly evolving into complex molecules in the gas and condensed phases with computer-tailored laser pulses, ${ }^{4,5}$ there are still many lessons to be learned from small molecules in the gas phase. Here we present a variation of the femtosecond three-pulse four-wave mixing (FWM) technique where the signal is spectrally dispersed. We find this method to be very useful to control and characterize the intramolecular dynamics occurring after laser excitation. In this study, we experimentally explore the role of different pulse sequences and optical-phase manipulation in controlling the population and coherence transfer in gas-phase $\mathrm{I}_{2}$. The two-dimensional (time and frequency) data provide phase information that cannot be obtained from similar techniques without spectral dispersion. Our experimental results and theoretical simulations demonstrate the role that pulse sequences and laser chirp can play in controlling intramolecular dynamics.

Here we concentrate on the controlled formation of groundand excited-state wave packets and the time-dependent polariza-

* To whom correspondence should be addressed: dantus@msu.edu.

$\dagger$ Affiliated with the Institute for Nuclear Sciences "VINCA", Belgrade, F. R. Yugoslavia.

$\doteqdot$ Permanent address: N. N. Semenov Institute of Chemical Physics, RAS, Moscow, Russia. tion that is observed when they are probed with a third laser pulse using three-pulse FWM. The goal of these experiments is to gain a deeper understanding about the role of chirped laser pulses in controlling chemical reactions. The results from this study are consistent with the experimental observation of wave packet modification (for example focusing) ${ }^{6-10}$ and enhancement in the yield of chemical reactions using chirped femtosecond pulses., ${ }^{3,11}$ Gas-phase $\mathrm{I}_{2}$ was chosen for the experimental demonstration of this technique. The visible $X^{1} \Sigma_{0+\mathrm{g}} \leftrightarrow \mathrm{B}^{3} \Pi_{0+\mathrm{u}}$ transition has been well characterized by frequency-resolved ${ }^{12-14}$ and time-resolved spectroscopy. ${ }^{15,16}$ The vibrational spacing is quite different for these two states, making their identification in the data a simple task.

An important step in the control of molecular excitation is the experimental characterization of the system being controlled. Conventional pump probe (PP) and FWM techniques give very high temporal resolution but give little or no spectral information. Some variations of the PP method have been shown to provide frequency resolution, among these are time-gated fluorescence techniques ${ }^{17-19}$ and PP-photoelectron spectroscopy. ${ }^{20,21}$ The additional frequency resolution helps one to obtain a more complete characterization of the system. The time gate used to obtain the spectral information determines the spectral resolution that can be achieved, limited by the uncertainty principle. Here we spectrally disperse the signal from the threepulse FWM. This signal contains a wealth of spectroscopic 
information. ${ }^{22}$ We have found that this information allows us to more fully characterize the system following different pulse sequences and different chirp values. In previous communications from our group, we have shown that the pulse sequence in three-pulse FWM allows one to form wave packets in the ground and/or excited states. ${ }^{22-24}$ This control of intramolecular dynamics was found to be quite general. Here we explore the relationship between the spectrally dispersed three-pulse FWM signal and the underlying dynamics. We then use this technique to characterize the effect of different pulse sequences and chirp values on the ground and the excited-state dynamics of the system.

A theoretical background is given in terms of the evolution of the density matrix. The formation of the wave packets in the ground and/or excited state by the first two pulses and the subsequent probing has been published elsewhere ${ }^{22,23}$ and is only mentioned here briefly. Here we concentrate on the spectroscopic information that is contained in the FWM signal and the connection to the density matrix. The Experimental Section briefly outlines the methodology used to obtain the twodimensional experimental data. Results are presented for various pulse sequences and pulse chirps. Theoretical calculations are found to be in excellent agreement with the experimental data. The Conclusions discuss critical parameters in the control of this system and highlight possible applications. Calculations are used to predict interesting behavior that can be achieved by particular sequence-chirp combinations.

\section{Theory}

The density matrix formulation is the preferred theoretical approach for the study of coherent multiwave mixing experiments. ${ }^{25,26}$ The diagonal blocks of the density matrix of a multilevel system involving several vibrational levels in the ground and excited electronic states represent properties within each state (see Figure 1a). In the diagonal blocks, the diagonal elements (designated as $\rho_{\mathrm{gg}}$ or $\rho_{\mathrm{ee}}$ in Figure 1a) are the population of each vibrational level, whereas the off-diagonal elements $\left(\rho_{\mathrm{gg}}{ }^{\prime}\right.$ or $\left.\rho_{\mathrm{ee}}\right)$ represent the coherence of the vibrational levels. The elements of the off-diagonal blocks $\left(\rho_{\mathrm{ge}}\right.$ or $\left.\rho_{\mathrm{eg}}\right)$ represent the vibronic coherence between the two electronic states. In the weak interaction limit, each field $E_{n}$ interacts linearly with the media producing a change $\rho^{(n)}$ to the initial density matrix. When $n$ is an odd number, $\rho^{(n)}$ contains the changes in the probability amplitude of the electronic couplings, whereas if $n$ is an even number, $\rho^{(n)}$ represents the changes in the population and the coherence of the vibrational levels within each electronic state.

The time evolution of the density matrix interacting with consecutive applied weak electric fields can be represented by double-sided Feynman diagrams. ${ }^{25,27}$ The diagrams relevant to our pulse sequence and phase-matching geometry are shown in Figure 1b. ${ }^{22,23,28}$ Here we only show the first interactions with electric fields $E_{\mathrm{a}}$ and $E_{\mathrm{b}}$ separated by a time delay $\tau_{\mathrm{ab}}$. Interaction with the third electric field $E_{\mathrm{c}}$ and the emitted signal $E_{\mathrm{s}}$ are not shown. The complex conjugate diagrams, mirror images of those shown in Figure 1b, lead to the FWM signal in a different phase-matching geometry and are not shown. The signal is a sum of all possible processes and arises from the contributions of $\rho_{\mathrm{g}^{\prime} \mathrm{g}}$ and $\rho_{\mathrm{ee}}$, which correspond to coherence in the ground and excited states, respectively, after the interaction with the first two pulses. Each process depends on the molecular dynamics of the corresponding electronic state; therefore, by enhancing one contribution with respect to the other, it is

\section{a) Multilevel Density Matrix}

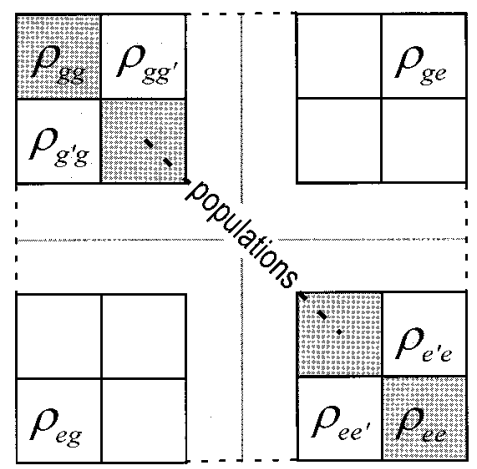

b) Double-Sided Feynman Diagrams

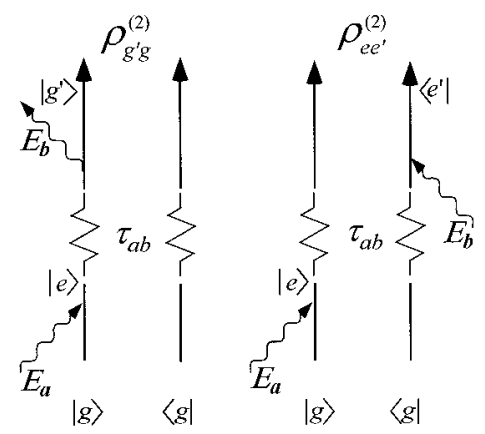

Figure 1. (a) Representation of the density matrix for a multilevel system composed of two electronic states each one with a manifold of vibrational levels. The indices $g, g^{\prime}$ range on the number of vibrational levels of the ground electronic state (in the present simulation $\mathrm{g}, \mathrm{g}^{\prime}=$ $1,2)$. The indices $\mathrm{e}, \mathrm{e}^{\prime}$ range on the number of vibrational levels of the excited electronic state (in the present simulation e, $\mathrm{e}^{\prime}=3,4$ ). The diagonal matrix elements, $\rho_{\mathrm{gg}}$ and $\rho_{\mathrm{ee}}$, represent the population of the vibrational levels (dark shade). The off-diagonal matrix elements of the diagonal blocks, $\rho_{\mathrm{gg}}$ and $\rho_{\mathrm{ee}}$, represent the vibrational coherence in the respective electronic state (light shade). The elements of the offdiagonal blocks, $\rho_{\mathrm{ge}}$ and $\rho_{\mathrm{eg}}$, represent the vibronic coherence between the electronic states. (b) Double-sided Feynman diagrams for the processes described in the text after the first two electric field interactions only.

possible to extract some information about the molecular dynamics of each state.

In a FWM process, the first two electric fields interfere and form time-dependent and static gratings in space. The third pulse probes these gratings, inducing a polarization in the medium that emits coherent radiation. When $\omega_{0}$, the carrier frequency of the pulses, is resonant with an electronic transition frequency, the interfering fields induce two successive dipole transitions, yielding a redistribution of the vibrational populations and coherences of the molecular ensemble, described in $\rho^{(2)}$. In this way the time-dependent matrix elements, $\rho_{\mathrm{g}_{\mathrm{g}} \mathrm{g}}^{(2)}(t)$ and $\rho_{\mathrm{e} e^{(}}^{(2)}(t)$, which oscillate with the vibrational frequencies of the ground and excited states, $\omega_{\mathrm{g}}$ and $\omega_{\mathrm{e}}$ respectively, describe the timedependent grating. The diagonal terms, $\rho_{\mathrm{gg}}^{(2)}$ and $\rho_{\mathrm{ee}}^{(2)}$, describe the static grating, which decays with $T_{1}$. Note in Figure $1 \mathrm{~b}$ that the diagram on the right contributes to $\rho_{\mathrm{ee}}^{(2)}$ while the one on the left contributes to $\rho_{\mathrm{g}^{\prime} \mathrm{g}}^{(2)}$. The transient FWM signal will be a maximum at the times when all molecules radiate in phase as long as the dephasing time of the excitation coherence, $T_{2}$, is longer than the duration of the pulses. ${ }^{24,29}$

In the present study, we apply the density matrix formulation for the interpretation of the signal obtained from chirped threepulse FWM experiments. Although we focus on the vibrational 
molecular dynamics, the generalization to rotational motion is straightforward. The theoretical analysis is based on a model that includes two electronic states with two vibrational levels each; the states are labeled as $|1\rangle,|2\rangle,|3\rangle$, and $|4\rangle$ where $g=1$, 2 and $\mathrm{e}=3,4$. The coherence relaxation time for $\mathrm{I}_{2}$ in the gas phase is much longer than the times considered here; therefore, no relaxation terms are included. Resonant experiments reduce the electronic manifold to the states that are coupled by the dipole electronic interaction. To simulate the observed molecular dynamics, we first use a model with two vibrational levels in each electronic state with an energy separation of $\Delta E_{\mathrm{g}}=\hbar \omega_{\mathrm{g}}$ or $\Delta E_{\mathrm{e}}=\hbar \omega_{\mathrm{e}}$. Although the effect of anharmonicity cannot be included in the formulation with this reduced model, an analytical solution to the optimal control problem is achieved. Unless otherwise noted, it is assumed that the initial populations are $\rho_{11}^{(0)}=\rho_{22}^{(0)}=0.5$ and $\rho_{33}^{(0)}=\rho_{44}^{(0)}=0$. We use $w=\rho_{11}^{(0)}-\rho_{22}^{(0)}$ to indicate a different initial population distribution.

In the present calculations and experiments, the three pulses are degenerate and designated as $E_{\mathrm{a}}, E_{\mathrm{b}}$, and $E_{\mathrm{c}}$. For the forward box arrangement described in ref 28, the FWM signal is emitted in two directions defined by the wave vectors $\mathbf{k}_{\mathrm{VE}}=\mathbf{k}_{\mathrm{a}}-\mathbf{k}_{\mathrm{b}}+$ $\mathbf{k}_{\mathrm{c}}$ and $\mathbf{k}_{\mathrm{E}}=-\mathbf{k}_{\mathrm{a}}+\mathbf{k}_{\mathrm{b}}+\mathbf{k}_{\mathrm{c}}$. The former applies to observation of a virtual echo and the latter to the conventional photon echo. ${ }^{25}$ Calculations and experimental results shown here apply to detection in the $\mathbf{k}_{\mathrm{VE}}$ direction.

In previous communications, ${ }^{22,23}$ we have shown that the simultaneous control of the population and vibrational coherence within each electronic state can be achieved by manipulating the time delay between the first two pulses, $\tau_{\mathrm{ab}}$. After the interaction with two unchirped pulses, the matrix elements of the ground and excited states are

$$
\begin{aligned}
& \rho_{\mathrm{gg}}^{(2)}=-\frac{A^{2}}{4} \cos \left(\frac{\omega_{\mathrm{e}} \tau_{\mathrm{ab}}}{2}\right) \cos \left(\left(\omega_{0} \pm \frac{\omega_{\mathrm{g}}}{2}\right) \tau_{\mathrm{ab}}-\left(\mathbf{k}_{\mathrm{a}}-\mathbf{k}_{\mathrm{b}}\right) \mathbf{x}\right) \\
& \rho_{\mathrm{gg}}^{(2)}(t)=\frac{A^{2}}{4} \cos \left(\frac{\omega_{\mathrm{e}} \tau_{\mathrm{ab}}}{2}\right) \mathrm{e}^{ \pm \mathrm{i} \omega_{\mathrm{g}}\left(t+(1 / 2) \tau_{\mathrm{ab}}\right)} \cos \left(\omega_{0} \tau_{\mathrm{ab}}-\left(\mathbf{k}_{\mathrm{a}}-\mathbf{k}_{\mathrm{b}}\right) \mathbf{x}\right) \\
& \rho_{\mathrm{ee}}^{(2)}=\frac{A^{2}}{4} \cos \left(\frac{\omega_{\mathrm{g}} \tau_{\mathrm{ab}}}{2}\right) \cos \left(\left(\omega_{0} \mp \frac{\omega_{\mathrm{e}}}{2}\right) \tau_{\mathrm{ab}}-\left(\mathbf{k}_{\mathrm{a}}-\mathbf{k}_{\mathrm{b}}\right) \mathbf{x}\right) \\
& \rho_{\mathrm{ee}^{\prime}}^{(2)}(t)=\frac{A^{2}}{4} \cos \left(\frac{\omega_{\mathrm{g}} \tau_{\mathrm{ab}}}{2}\right) \mathrm{e}^{ \pm \mathrm{i} \omega_{\mathrm{e}}\left(t+(1 / 2) \tau_{\mathrm{ab}}\right)} \cos \left(\omega_{0} \tau_{\mathrm{ab}}-\left(\mathbf{k}_{\mathrm{a}}-\mathbf{k}_{\mathrm{b}}\right) \mathbf{x}\right)
\end{aligned}
$$

where $A$ is the area under the pulse and the upper sign corresponds to the first combination of indices and vice versa for the lower sign. Setting $\tau_{\mathrm{ab}}=(n+1 / 2) \tau_{\mathrm{e}}$, where $\tau_{\mathrm{e}}=2 \pi / \omega_{\mathrm{e}}$, makes the ground state elements (eqs 1 and 2) equal to zero. For these values of $\tau_{\mathrm{ab}}$, we obtain a signal that is mostly characterized by the dynamics of the excited state. A similar observation can be made for $\tau_{\mathrm{ab}}=(n+1 / 2) \tau_{\mathrm{g}}$ with $\tau_{\mathrm{g}}=2 \pi / \omega_{\mathrm{g}}$. The vibrational coherence in the ground state corresponds to $\rho_{\mathrm{g}^{\prime} \mathrm{g}}^{(2)}$, whereas the vibrational coherence in the excited state corresponds to $\rho_{\mathrm{ee}^{\prime}}^{(2)}$.

When the first two pulses are equally chirped, the chirp is manifested in the vibrational coherence matrix elements $\rho_{\mathrm{gg}}^{(2)}(t)$ and $\rho_{\mathrm{ee}^{\prime}}^{(2)}(t)$ and is absent in $\rho_{\mathrm{gg}}^{(2)}$ and $\rho_{\mathrm{ee}}^{(2)}$. This is because the population matrix elements are obtained after a second-order process with two equal and opposite transition paths and the phase shift of the successive chirped pulses cancels out. ${ }^{30}$ When the transition paths are different, as for the coherence matrix elements, a net phase due to the chirped pulses arises. The modifications introduced by the chirped pulses to the spatial average of the density matrix elements are

$$
\begin{gathered}
\left\langle\left|\rho_{\mathrm{gg}}^{(2)}\right|^{2}\right\rangle \propto\left[1+\cos \left(\omega_{\mathrm{e}} \tau_{\mathrm{ab}}\right)\right] \\
\left\langle\left|\rho_{\mathrm{gg}}^{(2)}\right|^{2}\right\rangle \propto\left[1+\cos \left(\omega_{\mathrm{e}} \tau_{\mathrm{ab}}\right) \cos \left(\frac{\phi^{\prime \prime} \omega_{\mathrm{g}}{ }^{2}}{2}\right)\right] \\
\left\langle\left|\rho_{\mathrm{ee}}^{(2)}\right|^{2}\right\rangle \propto\left[1+\cos \left(\omega_{\mathrm{g}} \tau_{\mathrm{ab}}\right)\right] \\
\left\langle\left|\rho_{\mathrm{ee}}^{(2)}\right|^{2}\right\rangle \propto\left[1+\cos \left(\omega_{\mathrm{g}} \tau_{\mathrm{ab}}\right) \cos \left(\frac{\phi^{\prime \prime} \omega_{\mathrm{g}}{ }^{2}}{2}\right)\right]
\end{gathered}
$$

where the brackets denote the average with respect to coordinate x. $\phi^{\prime \prime}$ is the linear spectral chirp of the pulses obtained from the phase shift of the chirped electric fields, $\phi(\omega) \cong \phi\left(\omega_{0}\right)+$ $\left(\omega-\omega_{0}\right)^{2} \phi^{\prime \prime} / 2$ with $\phi^{\prime \prime}=\partial^{2} \phi / \partial \omega^{2}{ }^{31}$ Note that these expressions (eqs 5-8) are time independent and correspond to the amplitude of each component that contributes to the grating. The modulus square cancels the time-dependent terms in eqs 2 and 4.

After a time delay $\tau$, the third pulse in a FWM experiment probes the population and the vibrational coherence generated by the first two pulses. The resulting polarization can be represented by the third-order density matrix $\rho^{(3)}$ containing only the matrix elements that oscillate with the transition frequencies $\omega_{\text {eg }}=\left(E_{\mathrm{e}}-E_{\mathrm{g}}\right) / \hbar$ where $\mathrm{g}=1,2$ and $\mathrm{e}=3,4$. Therefore, the emitted light contains useful spectroscopic information about the system. The overall signal depends on $\rho^{(2)}=\rho^{(2) \mathrm{VE}}+\rho^{(2) \mathrm{E}}$, where the components that lead to signal in the $\mathbf{k}_{\mathrm{VE}}$ direction are separated from those that lead to signal in the $\mathbf{k}_{\mathrm{E}}$ direction. The intensity of the emitted light corresponding to each transition in the direction $\mathbf{k}_{\mathrm{VE}}$ as a function of $\tau$ is given by

$$
I_{\mathrm{eg}}^{\mathrm{VE}}(\tau) \propto\left|\rho_{\mathrm{ee}}^{(2) \mathrm{VE}}+\mathrm{e}^{\mathrm{i} \Phi} \rho_{\mathrm{ee}^{\prime}}^{(2) \mathrm{VE}}(\tau)-\rho_{\mathrm{gg}}^{(2) \mathrm{VE}}+\mathrm{e}^{-\mathrm{i} \Phi} \rho_{\mathrm{g}^{\prime} \mathrm{g}}^{(2) \mathrm{VE}}(\tau)\right|^{2}
$$

where $\Phi=\phi^{\prime \prime} \omega_{\mathrm{g}}{ }^{2} / 4$. Since the diagonal terms of the density matrix $\rho_{\mathrm{gg}}^{(2) \mathrm{VE}}$ and $\rho_{\mathrm{ee}}^{(2) \mathrm{VE}}$ are time independent, the time evolution of the spectral line intensity depends on the vibrational coherence matrix elements. Because these matrix elements oscillate with a vibrational frequency $\omega_{\mathrm{g}}$ or $\omega_{\mathrm{e}}$, eq 9 can be expressed as

$$
I_{\mathrm{eg}}^{\mathrm{VE}}(\tau) \propto I_{\mathrm{g}}^{\mathrm{VE}}(\tau)+I_{\mathrm{e}}^{\mathrm{VE}}(\tau)+I_{\mathrm{c}}^{\mathrm{VE}}(\tau)
$$

with

$$
I_{\mathrm{g}}^{\mathrm{VE}}(\tau) \propto\left\langle\left|\rho_{\mathrm{gg}}^{(2)}\right|^{2}\right\rangle\left[\cos \left(\omega_{\mathrm{g}} \tau\right)+1\right]
$$

and

$$
I_{\mathrm{e}}^{\mathrm{VE}}(\tau) \propto\left\langle\left|\rho_{\mathrm{ee}}^{(2)}\right|^{2}\right\rangle\left[\cos \left(\omega_{\mathrm{e}} \tau\right)+1\right]
$$

$I_{\mathrm{g}}^{\mathrm{VE}}$ and $I_{\mathrm{e}}^{\mathrm{VE}}$ represent the spectral intensity contributions that oscillate with the frequencies $\omega_{\mathrm{g}}$ and $\omega_{\mathrm{e}}$, respectively. $I_{\mathrm{c}}^{\mathrm{VE}}$ represents the cross terms that oscillate with a linear combination of both frequencies; if either $I_{\mathrm{g}}^{\mathrm{VE}}$ or $I_{\mathrm{e}}^{\mathrm{VE}}$ is zero, $I_{\mathrm{c}}^{\mathrm{VE}}$ is zero.

In Figure 2 we show the relationship between the amplitude of the averaged density matrix elements, as indicated in eqs $5-8$, and the spectral intensity, as shown in eq 9. The threedimensional diagrams of the averaged density matrix elements for the four-level system are shown at the top of the figure. The height represents the amplitude of $\left\langle\left|\rho_{i j}{ }^{(2)}\right|^{2}\right\rangle$. The spectral intensities as a function of time delay $\tau$ are shown in the spectrograms. The spectrograms show the contributions of the four transitions lines that correspond to the four-level system $\left(\lambda_{41}=4 \rightarrow 1, \lambda_{31}=3 \rightarrow 1, \lambda_{42}=4 \rightarrow 2\right.$, and $\left.\lambda_{32}=3 \rightarrow 2\right)$. Gaussian functions with a $\Delta \lambda / 2$ fwhm are used to simulate the experimental spectral resolution where $\Delta \lambda=\lambda_{41}-\lambda_{31}=\lambda_{42}$ 

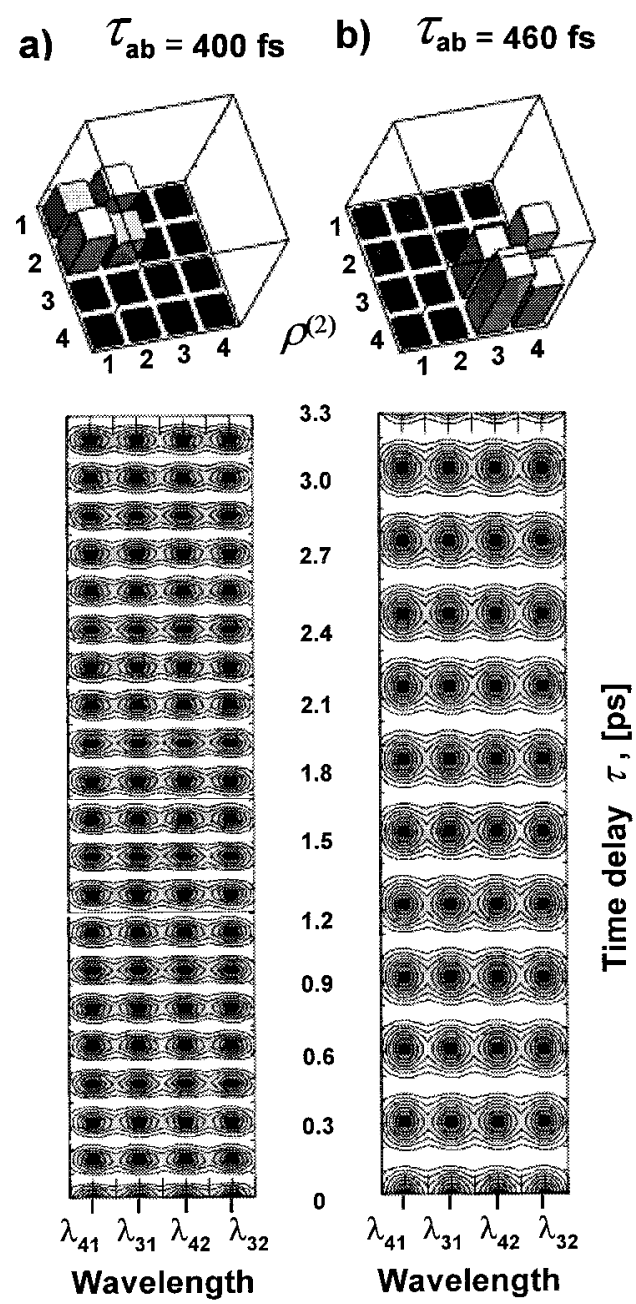

Figure 2. Simulations of spectrally dispersed FWM. At the top of the figure are diagrams of the averaged density matrix elements $\left\langle\left|\rho_{i j}^{(2)}\right|^{2}\right\rangle$ calculated with eqs $5-8$. The corresponding spectrograms calculated with eqs 9 are shown with wavelength plotted along the horizontal axis and time delay between the second and third pulses $(\tau)$ plotted along the vertical axis. The darker regions in the spectrograms correspond to higher signal intensity. For these calculations, the initial population distribution in the ground state $(w)$ was assumed to be zero and the pulses were unchirped. (a) The delay between first two pulses $\left(\tau_{\mathrm{ab}}\right)$ is $400 \mathrm{fs}$ corresponding to $5 / 2$ vibrational periods of the ground state, $\tau_{\mathrm{g}}$. The density matrix diagram for this pulse sequence (top left) shows population and coherence terms only in the upper diagonal block, corresponding to ground-state matrix elements alone. The spectrogram (left) clearly shows $160 \mathrm{fs}$ periods reflecting the vibration of the ground state $\left(\omega_{\mathrm{g}}\right)$. (b) For $\tau_{\mathrm{ab}}=460 \mathrm{fs}, 3 / 2$ vibrational periods of the excited state $\tau_{\mathrm{e}}$, only the matrix elements of the excited state (lower diagonal block) are present in diagram. In the corresponding spectrogram (right), the excited-state vibrations of $307 \mathrm{fs}$ are evident.

$-\lambda_{32}$. On the basis of eqs $5-8$, one can identify the time delay $\tau_{\mathrm{ab}}$ as a useful parameter in the design of pulse sequences that enhance or eliminate specific elements in the density matrix. In Figure 2, we show two extreme cases with either exclusive ground or exclusive excited-state dynamics. When $\tau_{\mathrm{ab}}=400$ $\mathrm{fs}=5 \tau_{\mathrm{g}} / 2$ (Figure $\left.2 \mathrm{a}\right)$, the spectral intensities oscillate with the vibrational frequency $\omega_{\mathrm{g}}$, with $\tau_{\mathrm{g}}=160 \mathrm{fs}$, consistent with the vibrational frequency of iodine in the ground state $\left(v^{\prime \prime}=2-4\right)$. The signal corresponds to the time evolution of the probability amplitude in the ground state because only the matrix elements $\rho_{\mathrm{gg}}^{(2)}$ and $\rho_{\mathrm{g}^{\prime} \mathrm{g}}^{(2)}(\tau)$ are different from zero (see the averaged density matrix diagram at the top of Figure 2a). When $\tau_{\mathrm{ab}}=$ $460 \mathrm{fs}=3 \tau_{\mathrm{e}} / 2$ (Figure $2 \mathrm{~b}$ ), the spectral line intensities oscillate with vibrational frequency $\omega_{\mathrm{e}}$, with $\tau_{\mathrm{e}}=307 \mathrm{fs}$, consistent with

$$
\tau_{\mathrm{ab}}=460 \mathrm{fs}
$$
a) $\phi^{\prime \prime}=1000 \mathrm{fs}^{2}$
b) $\phi^{\prime \prime}=4000 \mathrm{fs}^{2}$
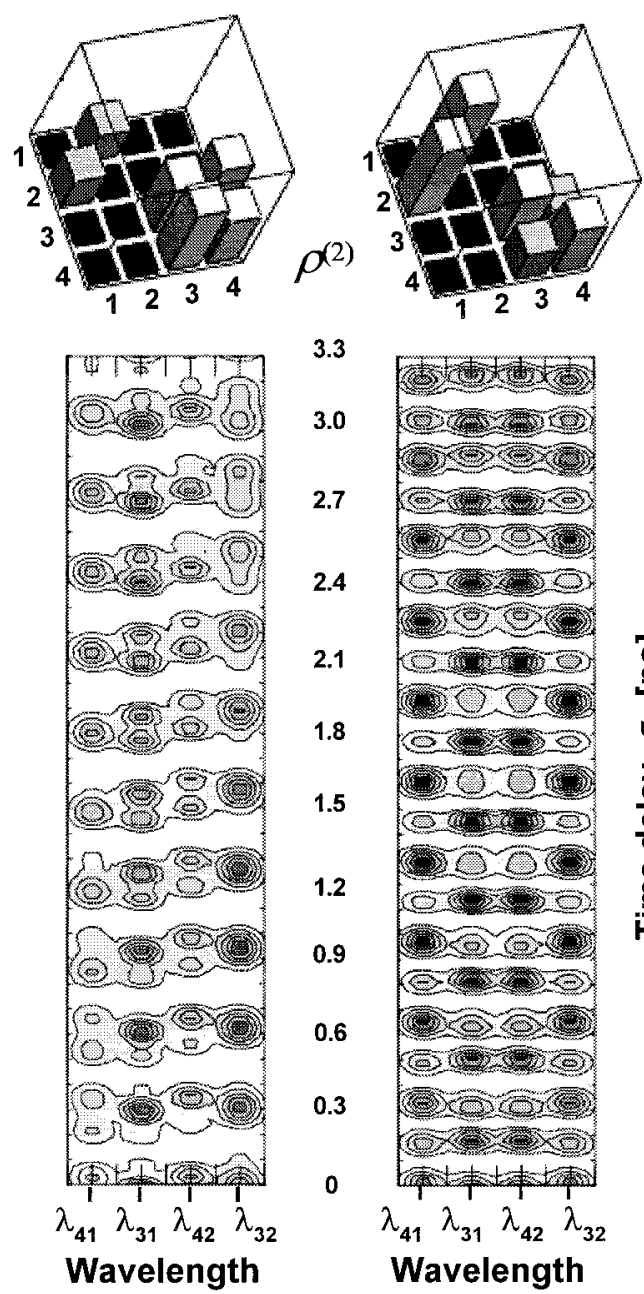

3.3

3.0

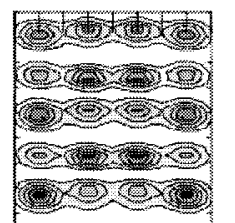

2.4
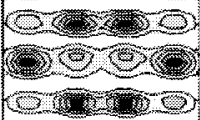

12) 018

erines

(8) 290

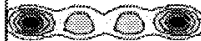

corse

(1) 680

$\Leftrightarrow$

(exs 610

(e) 8028

(e) 20,53

(c)

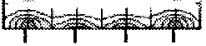

$\lambda_{41} \lambda_{31} \lambda_{42} \lambda_{32}$

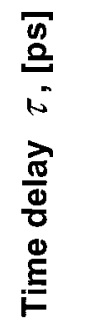

Wavelength

Figure 3. Simulations of spectrally dispersed FWM for $\tau_{\mathrm{ab}}=460 \mathrm{fs}$, $w=0$, and two values of laser pulse chirp. (a) For the small value of chirp $\left(\phi^{\prime \prime}=1000 \mathrm{fs}^{2}\right)$, the changes in the dynamics are obvious (compare to Figure 2b). These differences are expected, as seen in the density matrix diagram by the appearance of vibrational coherences in both the ground and excited states (upper and lower diagonal blocks). (b) It is possible to calculate the appropriate chirp where ground-state elements dominate the contribution to the signal even for $\tau_{\mathrm{ab}}=460 \mathrm{fs}$, where unchirped pulses result in seeing only excited-state dynamics. With $\phi^{\prime \prime}=4000 \mathrm{fs}^{2}$ (Figure 3b), $160 \mathrm{fs}$ oscillation periods appear in the simulation showing primarily ground-state dynamics.

the vibrational frequency of the $\mathrm{B}^{3} \Pi_{0+\mathrm{u}}$ state of iodine $\left(v^{\prime}=\right.$ 7-11). As can be seen from the averaged density matrix diagram (Figure $2 b$ top), only the elements of the excited state contribute to the signal in this case. Therefore, by changing the time delay between the first two pulses, it is possible to control the population and the vibrational coherence of the system.

The use of chirped pulses as an alternative way of controlling the intramolecular dynamics is explored in the simulations shown in Figure 3. The spectrograms along with the diagrams of the averaged density matrix elements correspond to time delay $\tau_{\mathrm{ab}}=460 \mathrm{fs}$ with chirped pulses having a spectral chirp $\phi^{\prime \prime}=$ $1000 \mathrm{fs}^{2}$ or $\phi^{\prime \prime} \omega_{\mathrm{g}}^{2} / 2=\pi / 4$ (Figure 3a) and $\phi^{\prime \prime}=4000 \mathrm{fs}^{2}$ or $\phi^{\prime \prime} \omega_{\mathrm{g}}{ }^{2} / 2=\pi$ (Figure $3 \mathrm{~b}$ ). The introduction of chirped pulses produces a signal that oscillates with both vibrational frequencies $\omega_{\mathrm{g}}$ and $\omega_{\mathrm{e}}$, confirmed by the presence of coherence vibrational 
terms belonging to both electronic states in the averaged density matrix diagram. When more than one oscillating frequency is present in the spectrogram, the spectral line intensity is slowly modulated in time with an approximate period of 3 ps. This effect can be attributed to the interference term, $I_{\mathrm{c}}^{\mathrm{VE}}(\tau)$, of the vibrational coherence terms from the different electronic states according to eq 10 .

Comparing the averaged density matrix elements distribution for different values of chirp for $\tau_{\mathrm{ab}}=460$ fs (Figures 2b top, $3 \mathrm{a}$ top, and $3 \mathrm{~b}$ top), we can see that the chirp does not affect the population terms but only the vibrational coherence terms, $\rho_{\mathrm{gg}}^{(2)}(t)$ and $\rho_{\mathrm{ee}^{\prime}}^{(2)}(t)$, in agreement with our previous discussion. Thus, the time dependence of the spectral signal can be manipulated through chirped electric fields by controlling the coherence vibrational terms of the density matrix after two pulses. Figure 3 highlights the importance of spectral dispersion; the intensity of the spectral features varies at different wavelengths due to the different phases involved. This information would not be available if the spectral information was integrated.

The density matrix elements after two pulses are the ensemble-averaged product of two time-dependent amplitudes, $\rho_{i j}^{(2)}(t)=\left\langle c_{i}(t) c_{j}(t)^{*}\right\rangle$, of the wave packet components in each electronic state. A change in the magnitude of $\rho_{\mathrm{gg}}^{(2)}(t)$ or $\rho_{\mathrm{ee}}^{(2)}(t)$ due to chirped pulses will correspond to a change in the relative phase of the wave packet components. This manipulation shapes the wave packet in each electronic state, allowing the focusing or spreading of the wave packet. ${ }^{6-8,10}$

When the initial population distribution is not limited to a single vibrational state, the density matrix elements represent the evolution of all levels in the system after successive laser pulses. A coherent coupling arises within each electronic state as a consequence of interaction with the first two pulses. For example, thermally populated vibrational levels in the sample prior to laser excitation lead to these coherences. The $\omega_{\mathrm{g}}$ dependence of eqs 3 and 4 is a manifestation of the coherence that arises from the initial population distribution. The density matrix formulation treats these coherences naturally without having to artificially add these contributions using phase coherent or incoherent sums of wavepackets. By taking only four levels, we were able to obtain analytical expressions for the spectrally dispersed FWM signal as a function of the control parameters $\tau_{\mathrm{ab}}$ and $\phi^{\prime \prime}$ using the density matrix formalism. Simulations based on these expressions are in close agreement with the experimental results. By increasing the number of the excited-state vibrational levels in the density matrix, we could explore the effect of anharmonicity and investigate the differences in the intensity features of the spectrally dispersed data (vide infra). These efforts are already underway. ${ }^{32}$ To give a physical illustration of the control mechanism, we carried out wave packet calculations consistent with the process depicted by the double-sided Feynman diagram shown in Figure 1b (lefthand side). Simulation of the other process (Figure $2 b$ righthand side), the probing step, and the resulting spectrally resolved signal by this method are more complicated and will be published elsewhere. ${ }^{33}$ In Figure 4 a, the initial wave packet $\Psi^{(0)}$ in $v^{\prime \prime}=2$ of the ground electronic state is excited by field $E_{\mathrm{a}}$ to the B excited state. The resulting excited-state wave packet $\Psi^{(1)}$ is allowed to evolve until field $E_{\mathrm{b}}$ is applied at time $\tau_{\mathrm{ab}}$. At time $\tau_{\mathrm{ab}}=460 \mathrm{fs}$ (see Figure $4 \mathrm{~b}$ ), the wave packet is located at the outer turning point; therefore, the Franck-Condon overlap with the optically resonant levels in the ground state is negligible. Note that the ground-state wavepacket in the figure has been magnified 10 times. The ground-state population $\left|\Psi^{(2)}\right|^{2}$ resulting from this process is very small for this value of $\tau_{\mathrm{ab}}$.

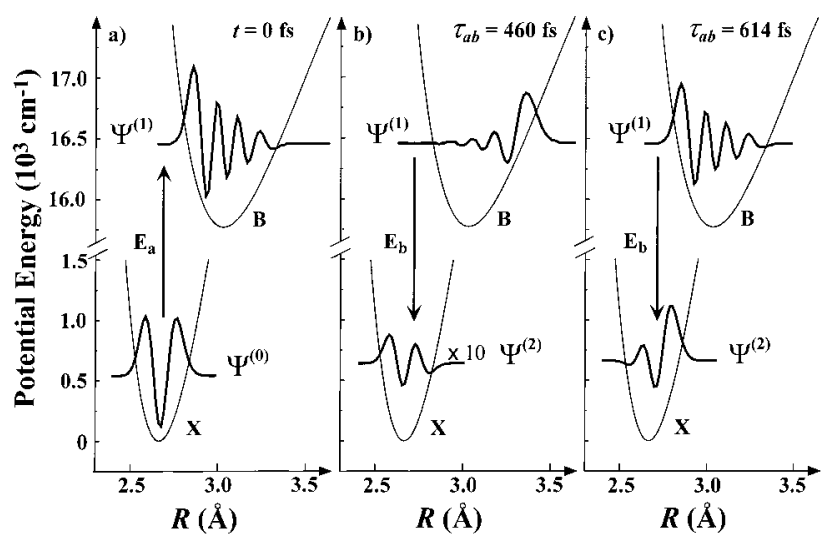

Figure 4. Wave packet calculations for the process described by the Feynman diagram labeled $\rho_{g^{\prime} g}^{(2)}$ in Figure $1 b$. In all cases only the real part of $\Psi$ is plotted. (a) Formation of the wave packet in the excited B state from an eigenstate $\left(v^{\prime \prime}=2\right)$ in the ground $\mathrm{X}$ state by field $E_{\mathrm{a}}$ at $t=0 \mathrm{fs}$. (b) The wave packet propagates and is shown after $460 \mathrm{fs}$ when the second field $E_{\mathrm{b}}$ is applied. At $\tau_{\mathrm{ab}}=460 \mathrm{fs}$, the wave packet in the B state is at its outer turning point, overlap with the $\mathrm{X}$ state is negligible, and population transfer to the ground state is minimal. Note that the magnitude of the wave packet in the ground state has been magnified 10 times. (c) When the second field interacts with the system after a delay $\tau_{\mathrm{ab}}=614 \mathrm{fs}$, the wave packet in the B state has returned to the inner turning point. The improved overlap with the optically resonant vibrational levels of the ground state allows for a greater transfer of population. These calculations are consistent with the observations of ground-state dynamics for $\tau_{\mathrm{ab}}=614 \mathrm{fs}$ and the absence of ground-state dynamics when $\tau_{\mathrm{ab}}=460 \mathrm{fs}$.

These calculations include all vibrational levels that can be optically excited by the femtosecond pulses centered at $620 \mathrm{~nm}$, namely $v^{\prime}=4-12$ and $v^{\prime \prime}=0-10$. When $\tau_{\mathrm{ab}}=614 \mathrm{fs}$ (see Figure $4 \mathrm{c}$ ), the wave packet is localized at the inner turning point, providing a good overlap with the optically resonant levels of the ground state and resulting in a significant ground-state population $(2-3$ orders of magnitude larger than that obtained for $\tau_{\mathrm{ab}}=460 \mathrm{fs}$ ). This behavior results from the period of oscillation of the wave packet in the excited state, $\tau_{\mathrm{e}}=307 \mathrm{fs}$. These wave packet calculations are useful to illustrate the position of the wave packet in each electronic potential as a function of time. However, an understanding of all the coherent processes that contribute to the FWM signal as gained from the density matrix formulation is essential. We are continuing to develop both formulations. The results from these efforts will be published elsewhere. ${ }^{32,33}$

\section{Experimental Section}

The experimental setup has been described previously. ${ }^{28}$ These experiments were conducted using 65 fs pulses (fwhm when transform-limited) centered at $620 \mathrm{~nm}$ with $8 \mathrm{~nm}$ (fwhm) spectral width. The chirp was measured by analyzing the pulses with a frequency-resolved optical gating (FROG). The laser was split into three beams, each with energy of $\sim 20 \mu \mathrm{J}$, which were arranged in the forward box configuration before being focused in the quartz sample cell containing neat iodine vapor at 140 ${ }^{\circ} \mathrm{C}$. The time delay between the first two pulses, $\tau_{\mathrm{ab}}$, was controlled by a manual translator and the time delay between the second and third pulse, $\tau$, was scanned in time to yield the FWM signal transients. The three-pulse FWM signal was collected by a spectrometer placed at the phase-matching condition $\mathbf{k}_{\mathrm{VE}}$ for different wavelengths within the 605-635 $\mathrm{nm}$ spectral range. The spectral acceptance of the spectrometer was set to $2 \mathrm{~nm}$. Narrower spectrometer slits did not seem to sharpen observed spectral features. Both FWM spectra and 
transients were measured with these same conditions. The spectra were obtained between 600 and $640 \mathrm{~nm}$ and averaged for 10 scans with 200 points per scan and 10 laser shots per point. Each transient was taken at 150 different time delays and averaged for 20 scans. At each time delay $\tau$, the signal was collected for 10 laser shots where the energy of each laser pulse was required to be within one standard deviation from the mean. These transients, collected at different wavelengths, were then combined to generate the spectrograms shown in the Results and Discussion.

\section{Results and Discussion}

A. Spectral Information in Femtosecond Three-Pulse Four-Wave Mixing. Most four-wave mixing experiments measure the intensity of the signal either as a function of excitation wavelength (frequency-resolved FWM) or as a function of time delay between laser pulses (time-resolved FWM). When the FWM measurements are performed with lasers that are not resonant with spectroscopic transitions (transient grating FWM), the polarization of the sample is shortlived and there is little spectroscopic information that can be obtained from spectrally resolving the FWM signal. ${ }^{28}$ For FWM measurements involving resonant excitation, the polarization can be sufficiently long-lived that it carries relevant spectroscopic information. ${ }^{22}$ This is particularly the case for gas-phase samples where the polarization can persist for tens to hundreds of picoseconds. ${ }^{24}$ Spectrally dispersed FWM measurements are not reserved only for long-lived polarization systems; for a radiative decay of $1 \mathrm{ps}$, one can expect to get $5 \mathrm{~cm}^{-1}$ spectral resolution. The spectral resolution in this case is almost 2 orders of magnitude better than the bandwidth of the femtosecond lasers used for the experiment.

In Figure 5, we show the spectrum of the transform-limited femtosecond laser used for these experiments (b) and the spectra of three-pulse FWM signal for two cases, (a) and (c). The experimental data (dots) were fit by Gaussian functions (black lines). The laser has a bandwidth of $210 \mathrm{~cm}^{-1}$. Trace (a) corresponds to the signal observed when all three beams coincide in time. This spectrum is very broad but shows some spectroscopic features with an average width of $80 \mathrm{~cm}^{-1}$. Trace (c) shows well-resolved spectroscopic transitions and corresponds to three-pulse FWM measurements in which $\tau_{\mathrm{ab}}=614$ fs and $\tau=614$ fs. The average width of these features is 50 $\mathrm{cm}^{-1}$. The main difference between traces (a) and (c) is that the signal in the former case includes multiple FWM pathways, many involving nonresonant processes that are short-lived, ${ }^{28}$ and the latter case arises from a long-lived polarization that decays in hundreds of picoseconds. ${ }^{24}$ The spectral features are much narrower than the laser but are significantly broadened by the rotational temperature of the sample (spectral congestion) and the spectral resolution of the spectrometer. No significant improvement in the spectral resolution was obtained when much narrower slits were used.

The spacing between the features in Figure $5 \mathrm{c}$ is $\Delta \lambda \cong 4 \mathrm{~nm}$ and corresponds to the vibrational spacing in the $\mathrm{B}{ }^{3} \Pi_{0+\mathrm{u}}$ state. ${ }^{34}$ The transitions arise from a polarization involving vibrational levels $v^{\prime}=7-11$ in the B state and vibrational levels $v^{\prime \prime}=$ $2-4$ in the $\mathrm{X}^{1} \Sigma_{0+\mathrm{g}}$ state. The vibrational levels with the highest probability of excitation are $v^{\prime}=9$ and 10 . Given the very small rotational constant of iodine molecules, there is a great deal of spectral congestion. Furthermore, the coincidental factor of 2 between the vibrational frequency of the ground state compared to that of the B state results in multiple band overlap. Transitions shifted to the red can be considered to be coherent Stokes Raman

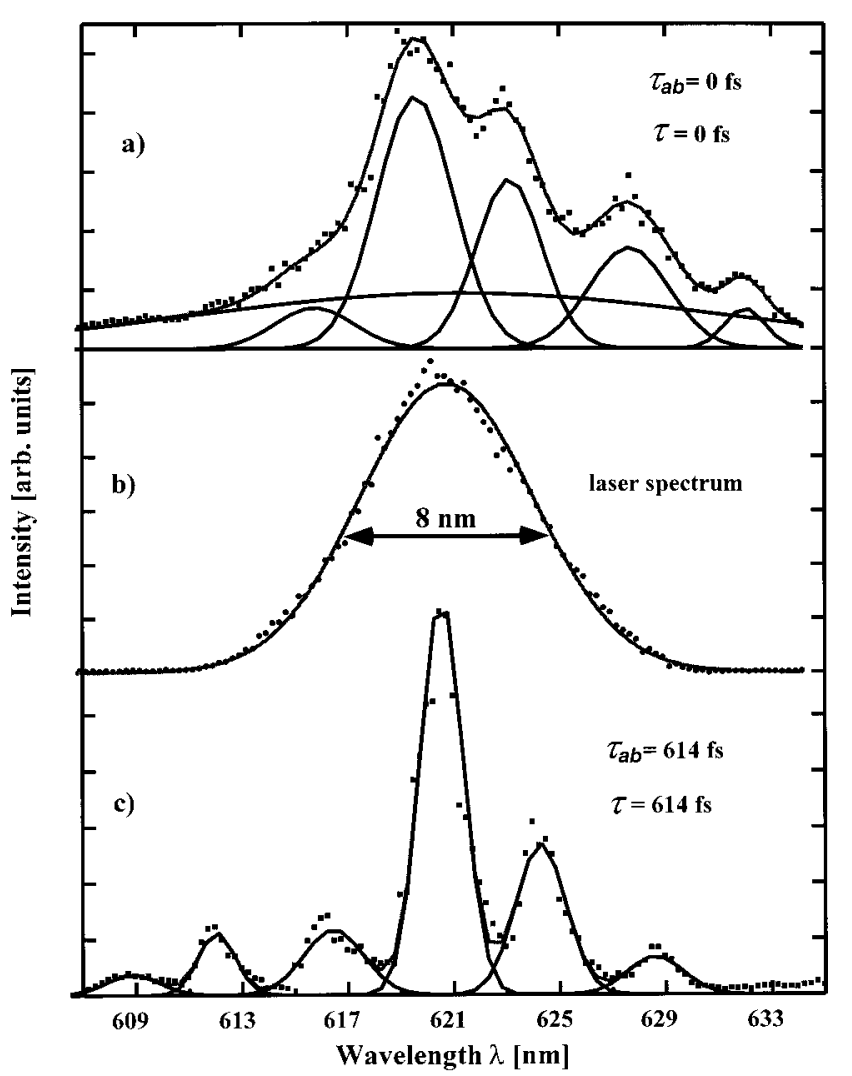

Figure 5. Experimental spectra (dots) and fits (lines) of the threepulse FWM signals and the transform-limited laser. (a) FWM signal observed when all three beams coincide in time ( $\left.\tau_{\mathrm{ab}}=0 \mathrm{fs} ; \tau=0 \mathrm{fs}\right)$. This spectrum is very broad but shows some spectroscopic transitions. The spectrum is fit with six Gaussian functions. (b) Spectrum of the unchirped femtosecond laser pulses used for these experiments. This profile is fit with a Gaussian function with width $8 \mathrm{~nm}$ (fwhm). (c) FWM signal observed when $\tau_{\mathrm{ab}}=614 \mathrm{fs}$ and $\tau=614 \mathrm{fs}$. This trace shows well-resolved spectroscopic transitions fit by six Gaussian functions. The spacing between the peaks in spectra (a) and (c) is 4 $\mathrm{nm}$, corresponding to the vibrational spacing of the B state of iodine.

scattering (CSRS) while those that are blue-shifted can be considered to be coherent anti-Stokes Raman scattering (CARS). ${ }^{35}$

The data in Figure 5 make it evident that spectral dispersion of the three-pulse FWM signal provides a new dimension with a wealth of information about the system. In the following sections, we explore how the spectra change as a function of time delay of the pulses involved in the three-pulse FWM process. We also explore the effects of chirped pulse excitation.

B. Two-Dimensional Three-Pulse FWM Measurements. The time-dependent intramolecular dynamics manifest themselves as changes in the intensity of the individual spectroscopic features. Therefore, the modulation of the signal is a manifestation of a time-dependent transition probability of the system. On the basis of these principles, it is not necessary to take a high-resolution spectrum at each time delay. The twodimensional data were obtained by taking transients at five to six different wavelengths and interpolating the rest of the spectroscopic information using multiple Gaussian functions in the same way that they were used in Figure 5 to fit the spectroscopic data. In all cases, spectra were taken at different time delays $\tau$ to check the two-dimensional data for wavelength and bandwidth consistency. Given that the data contain temporal as well as spectral information, it should be possible to invert and obtain the real space dynamics of the system in both the ground and the excited states. 


$$
\tau_{\mathrm{ab}}=0 \mathrm{fs} \quad \phi^{\prime \prime}=0 \mathrm{fs}^{2}
$$

a) Experiment

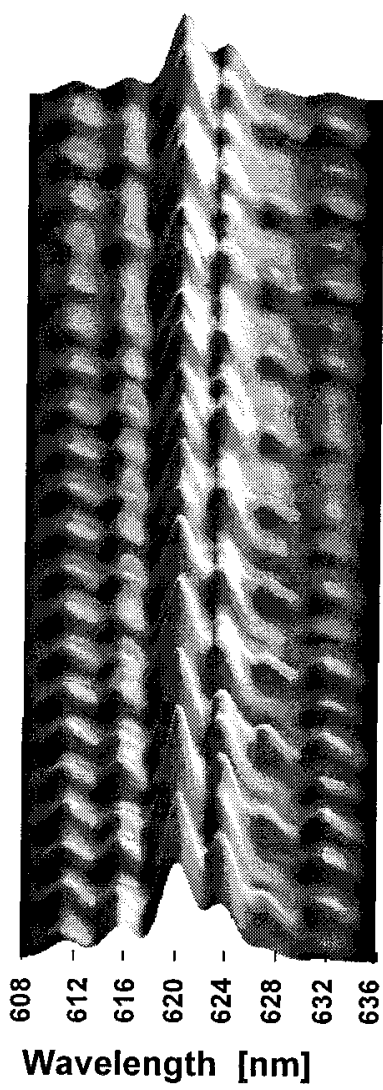

b) Experiment

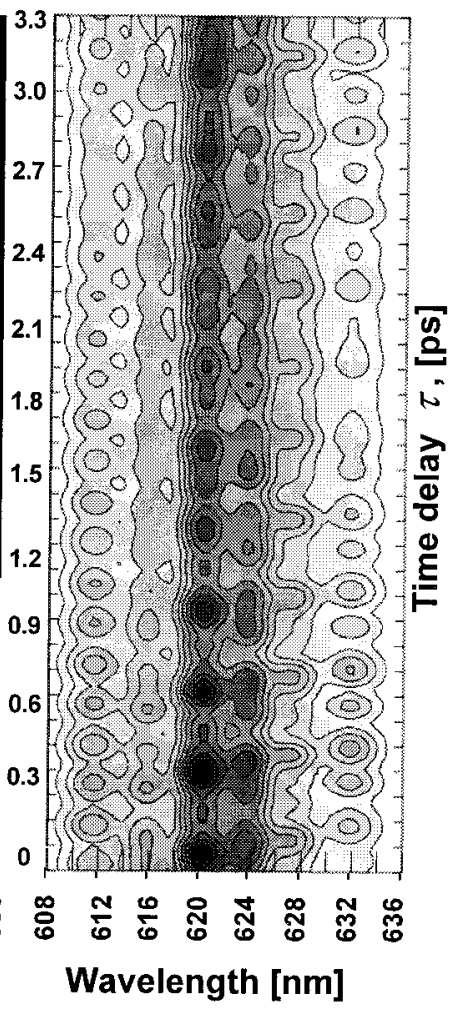

Figure 6. Spectrally dispersed experimental data for three-pulse FWM with $\tau_{\mathrm{ab}}=0 \mathrm{fs}$ and transform-limited pulses. Transients taken at six different wavelengths for 3.3 ps are represented as (a) a topographical plot and (b) a contour plot. As before, the darker areas in the contour plot correspond to the higher signal intensity whereas the topographical plot uses lighter shades to show higher intensity. Spectral profiles were approximated by Gaussian profiles (see Figure 5a,c) to generate the experimental spectrograms. Both ground- and excited-state dynamics are observed with these experimental conditions.

Figure 6 shows spectrally dispersed three-pulse FWM measurements carried out with $\tau_{\mathrm{ab}}=0 \mathrm{fs}$. Both plots present the two-dimensional experimental data. Figure 6 a shows the signal intensity in the third dimension, giving a topographic representation. Figure $6 \mathrm{~b}$ shows the same signal in a contour form that we find more useful for quantitative analysis. In the contour plot, darker shades correspond to higher intensity. The contour lines are spaced according to the square root of the signal amplitude. One of the important points to notice is that the spectroscopic features remain fixed in wavelength and one observes their change in intensity as a function of time delay $\tau$. This makes this technique different from wave packet imaging techniques such as those based on photoionization ${ }^{20}$ or Coulomb explosion. ${ }^{36,37}$ With those, one obtains the shape of the wave packet and its time evolution. In our measurements, the dynamics of the system are obtained from the change in intensity of every spectral region. The spectroscopy as well as the dynamics reveal the states involved; in the experiments discussed here, these would be the $\mathrm{X}$ and B states with $\omega_{\mathrm{g}}=208$ $\mathrm{cm}^{-1}$ and $\omega_{\mathrm{e}}=108 \mathrm{~cm}^{-1}\left(\tau_{\mathrm{g}}=160 \mathrm{fs}\right.$ and $\left.\tau_{\mathrm{e}}=307 \mathrm{fs}\right) .^{22,23}$

In Figure 6 the observed dynamics for wavelengths longer than $620 \mathrm{~nm}$ are a mixture of ground- and excited-state vibrational motion; however, the features with a period of 307 fs corresponding to the excited-state dynamics are more prevalent especially in the $628-632 \mathrm{~nm}$ region and after the first $1.5 \mathrm{ps}$. For wavelengths shorter than $620 \mathrm{~nm}$, the dynamics show a vibrational period of 160 fs that corresponds to the ground state. The observation of ground- and excited-state dynamics for FWM experiments with $\tau_{\mathrm{ab}}=0$ fs was reported first by Schmitt et al. ${ }^{38}$ Basically, the first two pulses create populations in both the ground and excited states. ${ }^{23,28}$ The third laser pulse probes the dynamics of both populations and the signal contains a mixture of each. Because of the FranckCondon factors between both of the electronic states involved, probing of the ground state will be enhanced for short wavelengths while probing of the excited state is enhanced for longer wavelengths. These enhancements result in the observed differences in the signal as seen in the experimental spectrograms. The detection wavelength has no effect on the intramolecular dynamics of the system.

With three-pulse four-wave mixing, one is able to prepare different pulse sequences that can be used to control the coherence and population, and therefore the observed dynamics of the system. ${ }^{22-24,28}$ Pulse sequences have been used in liquidphase FWM experiments to suppress vibrational mode contributions ${ }^{39}$ and to control the emission of signal by interfering with the echo and virtual echo components. ${ }^{40}$ In Figure 7 we show (a) experimental and (b) theoretical results for the case when $\tau_{\mathrm{ab}}=614 \mathrm{fs}$. This time delay maximizes the contribution from the ground state. The experimental data show fast oscillations with a period that corresponds to the vibrational frequency of the ground state. There is a small contribution from a population in the excited state that is apparent in the $628 \mathrm{~nm}$ region. The data show a phase shift of about $80 \mathrm{fs}$ when we compare the maxima of the spectra features for $612 \mathrm{~nm}$ to those at $628 \mathrm{~nm}$. This delay is probably due to the anharmonicity of the B state. Notice that the intensity remains high for the bluer wavelengths. The calculations, carried out for $w=0.33$, which corresponds to the expected Boltzmann distribution between the two vibrational levels in the ground state at $140{ }^{\circ} \mathrm{C}$, reproduce the observed dynamics, showing that higher intensities occur for shorter wavelengths and that some of the excited-state dynamics are observable for this pulse sequence. The calculations, based on a four-level density matrix, do not have the sufficient number of levels to reproduce the observed phase shift. The wave packet calculations shown in Figure 4 demonstrate that the observed ground-state dynamics arise from a wave packet that evolves in the B state for a time period $\tau_{\mathrm{ab}}$. During this evolution the anharmonicity of the B state causes the wave packet to spread. A density matrix calculation involving more excited-state vibrational levels would reproduce this observation as well.

When a pulse sequence is chosen with $\tau_{\mathrm{ab}}=460 \mathrm{fs}$, only the excited state is populated (see Figure 2). ${ }^{22-24}$ The experimental data, shown in Figure 8a, show very clear 307 fs oscillations that correspond to the vibrational period in the excited state for $v^{\prime}=7-11$. In this case there is no evidence of ground-state dynamics. These data can be contrasted with the data in Figure 7 where ground-state dynamics are dominant. The theoretical calculations, shown in Figure $8 \mathrm{~b}$ and carried out for $w=0.33$, are in excellent agreement with the experimental data. For this sequence, the intensity distribution across the spectral range is quite symmetric and shows no phase shift. Note that for this $\tau_{\mathrm{ab}}$, the calculations are independent of the initial population $(w)$ in the ground state.

C. Three-Pulse FWM with Chirped Laser Pulses. On the basis of the experimental data shown so far, it is clear that the spectrally dispersed three-pulse FWM data provide a wealth of 


$$
\tau_{\mathrm{ab}}=614 \mathrm{fs} \quad \phi^{\prime \prime}=0 \mathrm{fs}^{2}
$$

a) Experiment

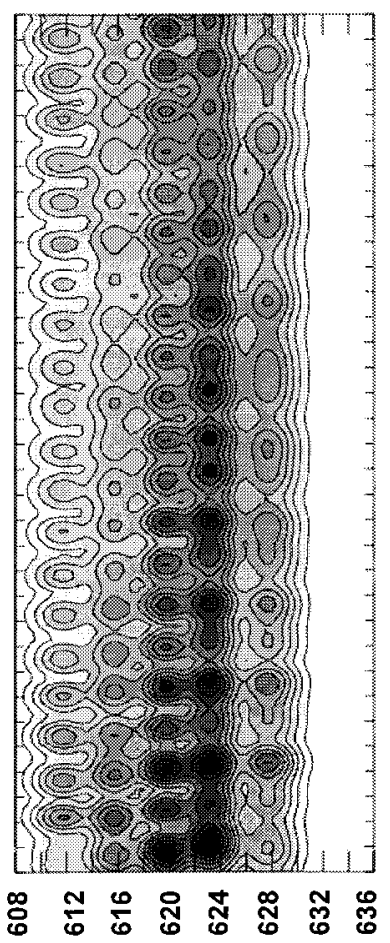

\section{Wavelength [nm]}

b) Theory

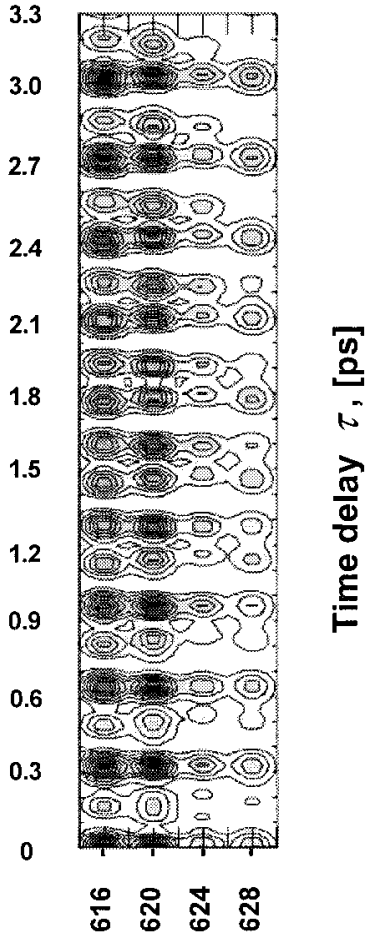

Wavelength [nm]
Figure 7. Spectrally dispersed experimental and theoretical spectrograms of three-pulse FWM for $\tau_{\mathrm{ab}}=614 \mathrm{fs}$ and $\phi^{\prime \prime}=0 \mathrm{fs}^{2}$. (a) The spectrogram shows the experimental transients taken at five different wavelengths where the spectral profiles were approximated by Gaussian profiles. For all wavelengths, the dominant feature is the $160 \mathrm{fs}$ vibrational motion, reflecting the ground-state dynamics. (b) The initial population distribution was taken to be $w=0.33$ for the calculation of the theoretical spectrogram of the experiment shown in (a). The $160 \mathrm{fs}$ vibrations from the ground-state dynamics are evident in both the theoretical and experimental spectrograms.

information about the intramolecular dynamics of a system, including information about the population distribution among the active electronic states. Here we explore the role that chirped laser pulses have on the excitation of molecular systems. Recent experiments where chirped laser pulses have been used to control the excitation of molecules and the yield of chemical reactions provide the motivation for this study. $3,10,11,41-44$

Using the pulse sequence with $\tau_{\mathrm{ab}}=460 \mathrm{fs}$ and fixing the time delay of the third pulse to $600 \mathrm{fs}$ give us a snapshot of the system in time. While keeping these two parameters fixed, we explore the effect of the pulse chirp on the dispersed spectra. The experimental data for this measurement are presented in Figure 9; a spline function is used to interpolate between the spectra obtained at the different chirp values. Notice that for zero chirp the spectrum is centered and is quite symmetrical, as discussed earlier. For positive chirps, we notice that the intensity shifts toward shorter wavelengths, while for negative chirps, the intensity shifts toward longer wavelengths. These shifts and their time dependence are discussed below. It is interesting to note that the highest signal intensities are observed for positive chirps. This observation is consistent with the fact that optimal pumping of the $\mathrm{B}$ state of iodine requires a positively chirped pulse. ${ }^{44-46}$

$$
\tau_{\mathrm{ab}}=460 \mathrm{fs} \quad \phi^{\prime \prime}=0 \mathrm{fs}^{2}
$$

a) Experiment

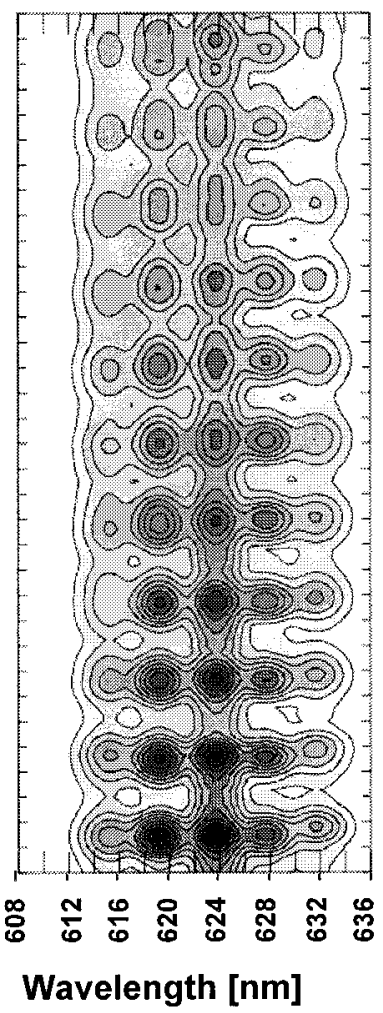

\section{b) Theory}

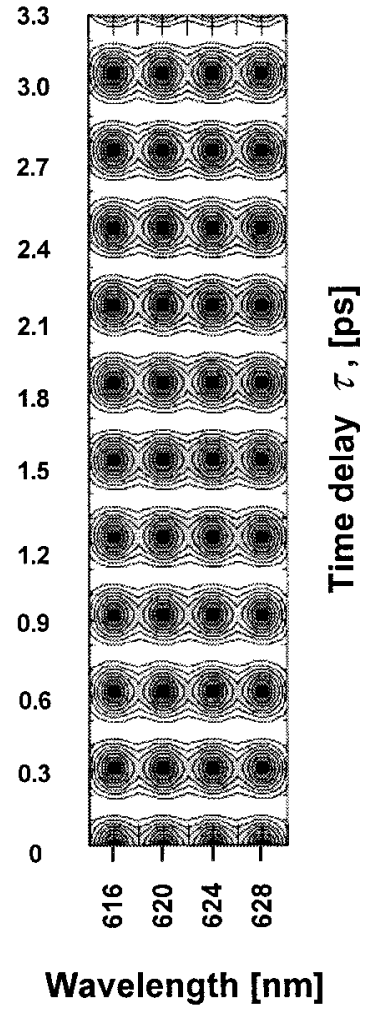

Figure 8. Spectrally dispersed experimental and theoretical spectrograms of three-pulse FWM for $\tau_{\mathrm{ab}}=460 \mathrm{fs}$ and $\phi^{\prime \prime}=0 \mathrm{fs}^{2}$. (a) The spectrogram generated from the transients taken at five different wavelengths shows only $307 \mathrm{fs}$ oscillations from the excited-state dynamics. There is no evidence of ground-state dynamics. (b) The simulation of the experiment again used $w=0.33$ for the calculation. The theoretical and experimental spectrograms are in excellent agreement with the 307 fs oscillations clearly evident in both.

In Figure 10 we present experimental and theoretical data for the two-dimensional three-pulse FWM where $\tau_{\mathrm{ab}}=460 \mathrm{fs}$ and the laser pulses are chirped to $3300 \mathrm{fs}^{2}$. By comparison of the experimental data in Figure 10a with the experimental data in Figure 8a obtained for the same pulse sequence, it is clear that the chirp has introduced ground-state dynamics into the observed signal. While the excited-state dynamics are still clearly observable, there are some regions showing oscillations with the $160 \mathrm{fs}$ characteristic of the ground state. There are spectral regions, for example, $620 \mathrm{~nm}$, where there is a clear doubling of the frequency, with every other beat having higher intensity. The theoretical calculations in Figure 10b, carried out with $w=0.33$ and $\phi^{\prime \prime}=3300 \mathrm{fs}^{2}$, are in excellent agreement with the experimental data. The calculations reproduce the shift of spectral intensity toward shorter wavelengths and the alternating intensity between the vibrational features.

Having learned to calculate the two-dimensional data for three-pulse FWM experiments allows us to explore different types of experiments. It is possible to find "critical chirp" values that accomplish certain tasks, for example, eliminate the groundor the excited-state dynamics in the signal. Here we would like to focus on two interesting cases. In Figure 11 we show simulations for the pulse sequence with $\tau_{\mathrm{ab}}=614 \mathrm{fs}$. When a relatively small chirp of $1000 \mathrm{fs}^{2}$ is used (a), we see that the ground-state contributions to the signal decrease (compare to 


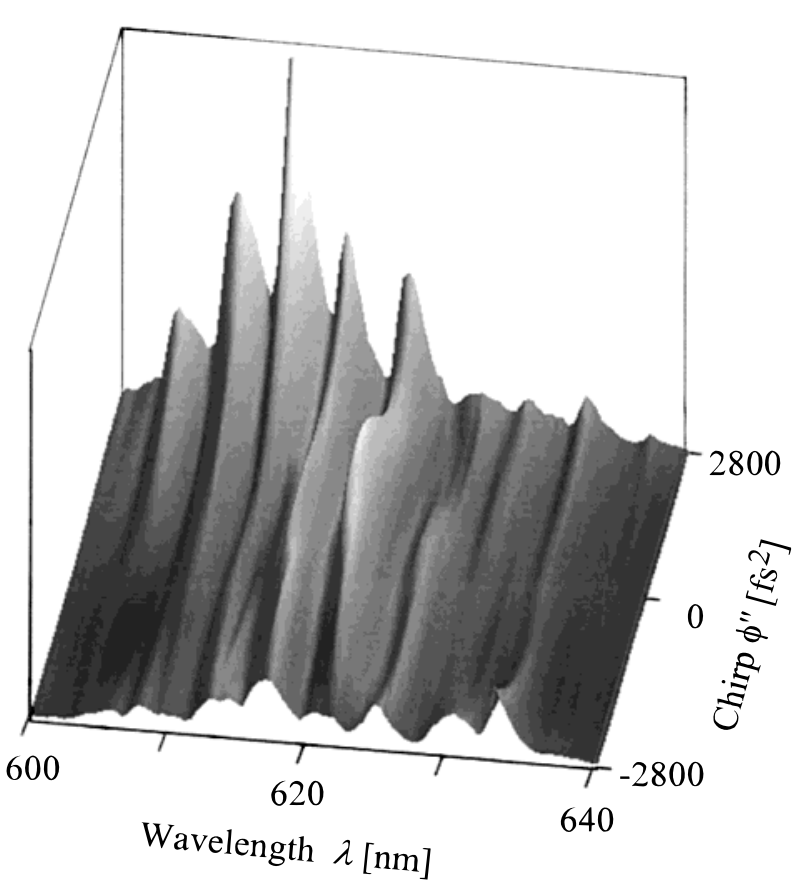

Figure 9. Three-dimensional representation of the experimental spectra taken using differently chirped pulses for the pulse sequence $\tau_{\mathrm{ab}}=460$ fs and $\tau=600 \mathrm{fs}$. Chirp values ranged from -2800 to $+2800 \mathrm{fs}^{2}$. Note the change in intensity toward shorter wavelengths as the chirp values increase from negative to positive values.

Figure 7b). The data show an apparent slope in which the spectroscopic features on the short wavelength side seem to reach a maximum earlier than those on the long wavelength side. This slope depends on the sign of the laser chirp. For negative chirps the opposite slope would be observed.

The second interesting case, shown in Figure 11b, corresponds to the same pulse sequence but with a critical chirp $\phi^{\prime \prime}=$ $\left(2 \pi / \omega_{\mathrm{g}}{ }^{2}\right)=4000 \mathrm{fs}^{2}$ (see eqs 6 and 8$)$. In this case, only $307 \mathrm{fs}$ dynamics are observed. The spectral intensity is still shifted toward shorter wavelengths, as expected for $w=0.33$ and $\tau_{\mathrm{ab}}$ $=614$ fs. For $w<0$, a red shift in spectral intensity would be observed. However, most notably is the phase of the observed oscillations across the different wavelengths. The average density matrix diagrams for both cases are also shown in Figure 11 (top). Notice that the laser chirp does not affect the population but the coherence between the states. For the case shown in Figure 11b, the ground-state coherence is no longer present and therefore not observed. As can be seen in Figure $7 b$, the spectrogram for $\tau_{\mathrm{ab}}=614 \mathrm{fs}$ is dominated by ground-state dynamics. When the critical chirp of $4000 \mathrm{fs}^{2}$ is introduced, the ground-state dynamics are completely eliminated (see Figure 11b). Similarly, the spectrogram for $\tau_{\mathrm{ab}}=460 \mathrm{fs}$ in Figure $8 \mathrm{~b}$ shows only excited-state dynamics. When the critical chirp of $4000 \mathrm{fs}^{2}$ is introduced, the dynamics are dominated by the ground state (see Figure 3b). From a theoretical point of view, changing the chirp $\left(\phi^{\prime \prime}\right)$ is as effective as changing the pulse sequence $\left(\tau_{\mathrm{ab}}\right)$ to control the observed molecular dynamics.

\section{Conclusions}

We have shown that the signal arising from time-resolved FWM measurements carries valuable information about the intramolecular dynamics of the system. The time delay between the first two pulses is a valuable parameter for controlling the population and coherence transfer. In all cases the theoretical

\section{$\tau_{\mathrm{ab}}=460 \mathrm{fs} \quad \phi^{\prime \prime}=3300 \mathrm{fs}^{2}$}

\section{a) Experiment}

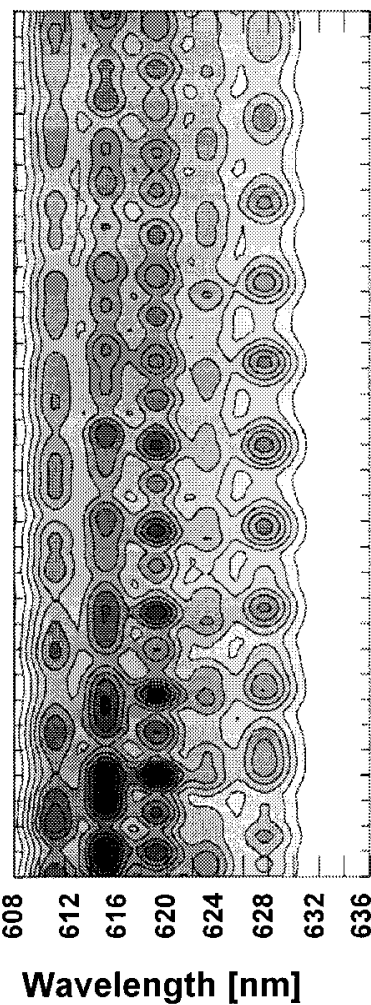

b) Theory

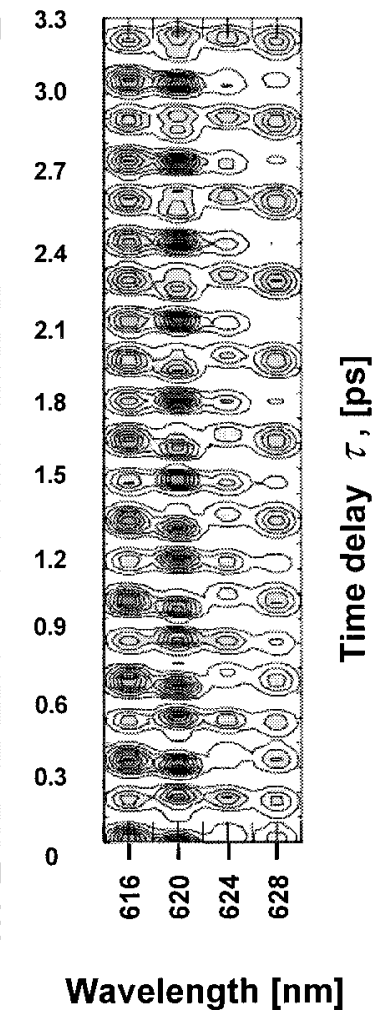

Figure 10. Spectrally dispersed experimental and theoretical spectrograms of three-pulse FWM for $\tau_{\mathrm{ab}}=460 \mathrm{fs}$ and $\phi^{\prime \prime}=3300 \mathrm{fs}^{2}$. (a) The experimental spectrogram was generated from transients obtained with five different wavelengths, as above. The signal observed for wavelengths $624 \mathrm{~nm}$ and shorter originates from mixed ground- and excited-state dynamics. It is clear when compared with Figure 8a that the addition of chirp alters the observed signal by introducing groundstate dynamics for shorter wavelengths. (b) The theoretical simulation, where $w=0.33$, shows good agreement with the experiment in both the dynamics features and the intensities of the beats.

simulations based on the formalism presented in the Theory were in excellent agreement with the experimental observations.

We have also explored the role of chirped pulse excitation. These measurements are of current interest as chirped pulses are being used to control the excitation process of molecules and the yield of laser-initiated chemical reactions. ${ }^{3,10,11,41-44}$ The data in Figure 10, obtained for $\phi^{\prime \prime}=3300 \mathrm{fs}^{2}$, show a contribution from the ground state that was absent in the dynamics obtained with unchirped pulses in Figure 8. We have shown that it is possible to find "critical chirp" values that can accomplish certain tasks, for example, introduce or eliminate ground-state dynamics in the signal. The theoretical simulations of the experiments using chirped pulses are in excellent agreement with the data, even though they are based on a small number of levels. All of our simulations are symmetric with respect to the sign of the laser chirp because only two vibrational levels are taken into account for each electronic state. Calculations that include multiple vibrational levels in the excited state would help to reproduce some of the finer features observed, such as the phase delay evident in the data for $\tau_{\mathrm{ab}}=614 \mathrm{fs}$ in Figure 7. From eqs 6 and 8 in the Theory, it is clear that the laser chirp $\left(\phi^{\prime \prime}\right)$ can be used as a parameter to control the 


$$
\tau_{\mathrm{ab}}=614 \mathrm{fs}
$$
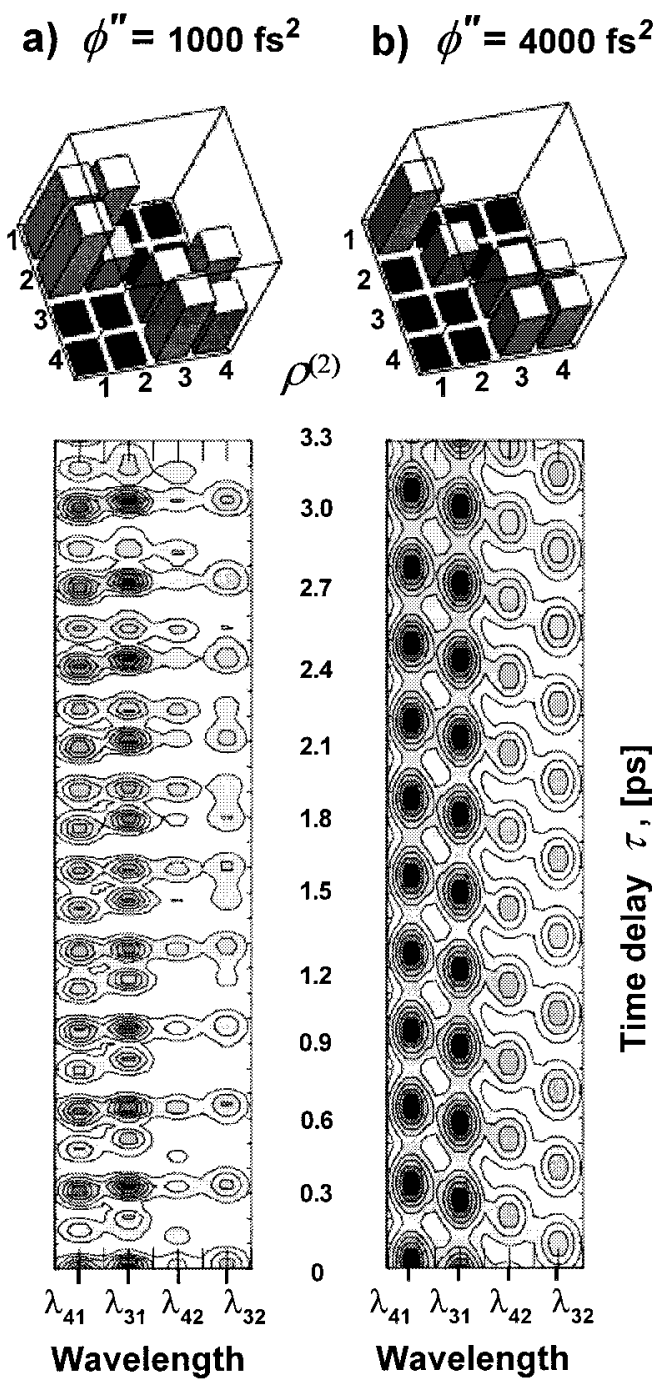

Figure 11. Diagrams of averaged density matrix elements $\left\langle\left|\rho_{i j}^{(2)}\right|^{2}\right\rangle$ and corresponding theoretical spectrograms for time delay $\tau_{\mathrm{ab}}=614$ fs and $w=0.33$ with $\phi^{\prime \prime}=1000 \mathrm{fs}^{2}$ and $\phi^{\prime \prime}=4000 \mathrm{fs}^{2}$. (a) For small chirps, the dynamics at shorter wavelengths reflect the ground state while higher wavelengths represent signal predominantly from the excited state. (b) When the pulses are chirped to the critical value, $\phi^{\prime \prime}$ $=\left(2 \pi / \omega_{\mathrm{g}}{ }^{2}\right)=4000 \mathrm{fs}^{2}$, the signal for all wavelengths reflects excitedstate dynamics only. Also notice that the density matrix diagram in this case shows population but no coherence in the ground state.

coherence, and in a similar way, the time delay $\left(\tau_{\mathrm{ab}}\right)$ can be used to control the coherence and population transfer (eqs 1-4).

The experimental data shown here illustrate the advantages of spectrally dispersed three-pulse FWM. For the case where $\tau_{\mathrm{ab}}=0 \mathrm{fs}$, one can see that longer wavelengths are dominated by excited-state dynamics while shorter wavelengths are dominated by ground-state dynamics (see Figure 6). This observation would not be possible with spectrally integrated data. For the case $\tau_{\mathrm{ab}}=614 \mathrm{fs}$, the data show a phase shift of about $80 \mathrm{fs}$ across the spectrum (see Figure 7). This shift is caused by the anharmonicity in the excited state. Therefore, the data contain phase information that is important in understanding the underlying intramolecular dynamics. Spectrally integrated data would have shown washed-out features. When chirped pulses are used, important spectral shifts occur. Figure 9 shows that for positive chirps the intensity shifts to shorter wavelengths and the signal intensity increases. Negatively chirped pulses give a broader spectrum that is shifted toward longer wavelengths. The spectral dispersion reveals important phase information about the system that is not available in spectrally integrated data.

The experiments presented here show control of population and coherence using multiple pulse sequences and chirps. The theoretically calculated effects indicate absolute control in the population and coherence transfer. Very large effects are shown here experimentally. The above examples demonstrate both experimentally and theoretically that the chirp controls the coherence but not the population transfer in this system. It is important to note that all the laser pulses are equally chirped and only two electronic states participate in the observed dynamics. When the first two laser pulses are chirped differently (magnitude and/or sign), one can control the population transfer. ${ }^{30}$ When the system contains a reactive pathway, the coherence can be used to enhance or decrease the probability of the system for following that pathway. The coherence determines the dynamics of the system. In multidimensional systems, one may want to control the yield of different pathways or affect the rate of intramolecular vibrational relaxation. This type of control, where the phase of the quantum mechanical superpositions are manipulated by the optical phases of the pulses to determine the outcome of chemical reactions, results in true control of final population transfer from reactant to product. Alternatively, the first two pulses can be used to prepare the system in a specific superposition of states that maximizes the yield of a specific product in a chemical reaction upon interaction with the third pulse.

So far we have only discussed sequences of linearly chirped pulses. With phase masks one can achieve quadratic and higher order chirps. ${ }^{47-49}$ One can also tailor the pulses into very complex electric fields, which have been used to achieve different product yields in chemical reactions. ${ }^{4}$ At present we find that much remains to be learned from experiments using multiple pulse sequences and linear chirps. Furthermore, these experiments have a transparent relationship with the molecular Hamiltonian. On the basis of these observations, we consider three-pulse FWM to be a powerful tool for probing intramolecular dynamics and for achieving laser control of chemical reactions.

Note Added in Proof: Book and Scherer ${ }^{50}$ have recently published wavelength-resolved FWM measurements on DTTCI dye in solution. Our findings are consistent with their conclusion that valuable information can be obtained from spectrally resolving the FWM signal.

Acknowledgment. This research was partially funded by a grant from the National Science Foundation (CHE-9812584). M.D. is a Lucille and David Packard Science and Engineering Fellow, a Camille Dreyfus Teacher-Scholar, and an Alfred P. Sloan Fellow. E.J.B. is supported by a National Science Foundation Graduate Fellowship. I.P. gratefully acknowledges a James L. Dye Endowment Fellowship. It is a pleasure to acknowledge fruitful discussions with Y. J. Yan.

\section{References and Notes}

(1) Chemical Reactions and Their Control on the Femtosecond Time scale; Gaspard, P., Burghardt, I., Eds.; Advances in Chemical Physics; Wiley: New York, 1997; Vol. 101.

(2) Gordon, R. J.; Rice, S. A. Annu. Rev. Phys. Chem. 1997, 48, 601

(3) Pastirk, I.; Brown, E. J.; Zhang, Q.; Dantus, M. J. Chem. Phys. 1998, 108,4375 .

(4) Assion, A.; Baumert, T.; Bergt, M.; Brixner, T.; Kiefer, B.; Seyfried, V.; Strehle, M.; Gerber, G. Science 1998, 282, 919.

(5) Bardeen, C. J.; Yakovlev, V. V.; Wilson, K. R.; Carpenter, S. D.; Weber, P. M.; Warren, W. S. Chem. Phys. Lett. 1997, 280, 151. 
(6) Kohler, B.; Krause, J. L.; Raksi, F.; Wilson, K. R.; Yakovlev, V. V.; Whitnell, R. M.; Yan, Y. J. Acc. Chem. Res. 1995, 28, 133.

(7) Kohler, B.; Yakovlev, V. V.; Che, J.; Krause, J. L.; Messina, M.; Wilson, K. R.; Schwentener, N.; Whitnell, R. M.; Yan, Y. J. Phys. Rev Lett. 1995, 74, 3360.

(8) Bardeen, C. J.; Che, J.; Wilson, K. R.; Yakovlev, V.; Apkarian, V. A.; Martens, C. C.; Zadoyan, R.; Kohler, B.; Messin, M. J. Chem. Phys. 1997, 106, 8486.

(9) Uberna, R.; Khalil, M.; Williams, R. M.; Papanicola, J. M.; Leone,

S. R. J. Chem. Phys. 1998, 108, 9259.

(10) Lozovoy, V. V.; Antipin, S. A.; Gostev, F. E.; Titov, A. A.; Tovbin, D. G.; Sarkisov, O. M.; Vetchinkin, A. S.; Umanskii, S. Y. Chem. Phys. Lett. 1998, 284, 221.

(11) Bardeen, C. J.; Che, J.; Wilson, K. R.; Yakovlev, V. V.; Cong, P.; Kohler, B.; Krause, J. L.; Messina, M. J. Phys. Chem. A 1997, 101, 3815.

(12) Steinfeld, J. I.; Zare, R. N.; Jones, L.; Lesk, M.; Klemper, W. J.

Chem. Phys. 1965, 42, 25.

(13) LeRoy, R. J. J. Chem. Phys. 1970, 52, 2683.

(14) Tellinghuisen, J.; McKeever, M. R.; Sur, A. J. Mol. Spectrosc. 1980, $74,225$.

(15) Gruebele, M.; Roberts, G.; Dantus, M.; Bowman, R. M.; Zewail,

A. H. Chem. Phys. Lett. 1990, 166, 459.

(16) Dantus, M.; Bowman, R. M.; Zewail, A. H. Nature 1990, 343, 737.

(17) Dunn, T. J.; Sweetser, J. N.; Walmsley, I. A. Phys. Rev. Lett. 1993 70,3388 .

(18) Shapiro, M. J. Chem. Phys. 1995, 103, 1748.

(19) Shapiro, M. J. Phys. Chem. 1996, 100, 7859.

(20) Assion, A.; Baumert, T.; Geisler, M.; Seyfried, V.; Gerber, G. Eur. Phys. J. D 1998, 4, 145.

(21) Blanchet, V.; Stolow, A. J. Chem. Phys. 1998, 108, 4371.

(22) Pastirk, I.; Brown, E. J.; Grimberg, B. I.; Lozovoy, V. V.; Dantus, M. Faraday Discuss. 1999, 113.

(23) Brown, E. J.; Pastirk, I.; Grimberg, B. I.; Lozovoy, V. V.; Dantus, M. J. Chem. Phys. 1999, 111, 3779.

(24) Lozovoy, V. V.; Pastirk, I.; Dantus, M. J. Chem. Phys., submitted for publication.

(25) Mukamel, S. Principles of Nonlinear Optical Spectroscopy; Oxford: New York, 1995.

(26) Boyd, R. W. Nonlinear Optics; Academic Press: Boston, 1992. (27) Shen, Y. R. The Principle of Nonlinear Optics; Wiley: New York, 1984.

(28) Brown, E. J.; Zhang, Q.; Dantus, M. J. Chem. Phys. 1999, 110, 5772 .
(29) Weiner, A. M.; Silvestri, S. D.; Ippen, E. P. J. Opt. Soc. Am. B $1985,2,654$

(30) Lozovoy, V. V.; Grimberg, B. I.; Brown, E. J.; Pastirk, I.; Dantus, M. J. Raman Spectrosc., in press.

(31) Femtosecond Laser Pulses: Principles and Experiments; Rullière, C., Ed.; Springer: Berlin, 1998.

(32) Grimberg, B. I.; Lozovoy, V. V.; Mukamel, S.; Dantus, M. Manuscript in preparation.

(33) Grimberg, B. I.; Lozovoy, V. V.; Dantus, M. Manuscript in preparation.

(34) Barrow, R. F.; Yee, K. K. J. Chem. Soc., Faraday Trans. 21973 69,684 .

(35) Schmitt, M.; Knopp, G.; Materny, A.; Kiefer, W. Chem. Phys. Lett. 1997, 280, 339 .

(36) Stapfeldt, H.; Constant, E.; Corkum, P. B. Phys. Rev. Lett. 1995, 74,3780 .

(37) Stapfeldt, H.; Constant, E.; Sakai, H.; Corkum, P. B. Phys. Rev. A 1998, 58,426

(38) Schmitt, M.; Knopp, G.; Materny, A.; Kiefer, W. Chem. Phys. Lett. 1997, 270,9 .

(39) Bardeen, C. J.; Shank, C. V. Chem. Phys. Lett. 1993, 203, 535.

(40) Pshenichnikov, M. S.; Boeij, W. P. d.; Wiersma, D. A. Phys. Rev. Lett. 1996, 76, 4701

(41) Yakovlev, V.; Bardeen, C. J.; Che, J.; Cao, J.; Wilson, K. R. J. Chem. Phys. 1998, 108, 2309.

(42) Bardeen, C. J.; Yakovlev, V.; Squier, J. A.; Wilson, K. R. J. Am Chem. Soc. 1998, 120, 13023.

(43) Buist, A. H.; Muller, M.; Ghauharali, R. I.; Brakenhoff, G. J.; Squier, J. A.; Bardeen, C. J.; Yakovlev, V. V.; Wilson, K. R. Opt. Lett. 1999, 24, 244.

(44) Zadoyan, R.; Schwenter, N.; Apkarian, V. A. Chem. Phys. 1998, $233,353$.

(45) Shen, Z. W.; Yan, Y. J.; Cheng, J. X.; Shuang, F.; Zhao, Y.; He, G. Z. J. Chem. Phys. 1999, 110, 7192 .

(46) Cao, J. S.; Bardeen, C. J.; Wilson, K. R. Phys. Rev. Lett. 1998, 80, 1406.

(47) Weiner, A. M.; Leaird, D. E.; Patel, J. S.; Wullert, J. R. IEEE J. Quantum Elect. 1992, 28, 908

(48) Hillegas, C. W.; Tull, J. X.; Goswami, D.; Strickland, D.; Warren, W. S. Opt. Lett. 1994, 19, 737 .

(49) Wefers, M. M.; Nelson, K. A.; Weiner, A. M. Opt. Lett. 1996, 21 746.

(50) Book, L. D.; Scherer, N. F. J. Chem. Phys. 1999, 111, 792. 